\title{
Grape Seed Proanthocyanidin Extract Down-Regulates Autophagic Proteins (LC3II and Beclin-1) in a Mouse Model of Neonatal Hypoxic-Ischemic Brain Injury
}

\author{
Li Luo ${ }^{1}$, Xing Tu ${ }^{1}$, Wei Liu ${ }^{2}$, Xuexia Zhang ${ }^{3}$, Yilin Liu ${ }^{2}$, Wenyan Zhao², Junhua Yang ${ }^{1}$, \\ Mengxia Wang ${ }^{4}$, , Jing Liu ${ }^{1, *}$ \\ ${ }^{1}$ School of Biosciences \& Biopharmaceutics, Guangdong Pharmaceutical University, Guangzhou, People's Republic of China \\ ${ }^{2}$ School of Clinical Medicine, the First Affiliated Hospital of Guangdong Pharmaceutical University, Guangzhou, People's Republic of China \\ ${ }^{3}$ Department of Anesthesiology, the Fifth Affiliated Hospital, Sun Yat-Sen University, Zhuhai, People's Republic of China \\ ${ }^{4}$ Intensive Care Unit, Guangdong No. 2 Provincial People's Hospital, Guangzhou, People's Republic of China
}

Email address:

liulq1227@163.com (Jing Liu), gracemengxia@hotmail.com (Mengxia Wang)

${ }^{*}$ Corresponding author

\section{To cite this article:}

Li Luo, Xing Tu, Wei Liu, Xuexia Zhang, Yilin Liu, Wenyan Zhao, Junhua Yang, Mengxia Wang, Jing Liu. Grape Seed Proanthocyanidin Extract Down-Regulates Autophagic Proteins (LC3II and Beclin-1) in a Mouse Model of Neonatal Hypoxic-Ischemic Brain Injury. Clinical Medicine Research. Vol. 8, No. 1, 2019, pp. 16-20. doi: 10.11648/j.cmr.20190801.13

Received: January 31, 2019; Accepted: March 11, 2019; Published: March 25, 2019

\begin{abstract}
Neonatal hypoxic-ischemic encephalopathy (HIE) is a severe brain disease that often leads to death or irreversible sequelae. The aim of this study was to determine the effect of a naturally active drug (grape seed proanthocyanidin extract [GSPE]) on the expression of autophagic proteins in a mouse model of neonatal hypoxic-ischemic brain injury. In this study immunofluorescence staining and Western blotting were used to detect the expression of autophagy markers (LC3II and beclin1) in the brains of neonatal mice with hypoxic-ischemic brain injuries after GSPE administration. Our study showed that GSPE pre-treatment down-regulates LC3II and beclin-1 expression, thus GSPE may be a potential drug for the treatment of HIE.
\end{abstract}

Keywords: Grape Seed Proanthocyanidins Extract, Hypoxic-Ischemic Brain Injury, Autophagy

\section{Introduction}

Hypoxic-ischemic encephalopathy (HIE) is a severe brain injury with various causes in the perinatal period. HIE is often accompanied by high mortality and disability rates $[1,2]$. According to current statistics, neonatal asphyxia occurs in 2-4 of 10,000 full-term newborns, and $20 \%-50 \%$ die as a result of HIE during the neonatal period. The survivors often have serious sequelae, such as epilepsy, cerebral palsy, and mental retardation [3]. Children with HIE often undergo hypothermia therapy [4]. Hypothermia therapy has a good curative effect in patients with mild-to-moderate brain injury, but hypothermia therapy has little effect in patients with severe HIE brain injuries [5]. Therefore, there is an urgent need to identify a targeted drug to treat HIE.

Grape seed proanthocyanidin extract (GSPE) is a naturally active drug that is formed by the condensation of catechins and/or epicatechins $[6,7]$. GSPE is widely available and can be extracted from a variety of plants [8, 9], such as grape seed, pineal peel, and apples. GSPE has been shown to have good efficacy in cardiovascular and neurologic disease models [10]. The results of our preliminary studies have demonstrated that GSPE significantly improves cerebral infarction volume and neurobehavior in a murine model of HIE, and GSPE pre-treatment attenuates apoptosis through the bax/bcl2-cleaved caspase 3 signaling pathway. It is well-known that autophagy and apoptosis are the main ways causing cell death, and the two processes are inseparable; however, there have been no studies which have shown that the changes in autophagy levels after GSPE pre-treatment in the HIE model. In the current study we designed experimental tests to detect changes in autophagy-related proteins in the presence of a HI injury. Based on the previous work, we confirmed that GSPE can alleviate neurologic damage of HIE through autophagy. 


\section{Method}

\subsection{Establishment of the HIE Model and GSPE Administration}

We selected C57 mice, 7-9 days of age, to establish the HIE model [11]. The pups were male or female. All experimental animals were purchased from the Medical Experimental Animal Center of Sun Yat-sen University. The study protocol was reviewed by the Ethics Committee of Guangdong Pharmaceutical University and satisfied the guidelines of the Chinese Council on Animal Care. During the experiments, we strived to minimize pain and the number of animals used.

We use the modified Rice-Vannucci method to establish the HIE disease model. The specific procedure was as follows. Pups were anesthetized with $3 \%$ isoflurane until they lost consciousness. We used electrocautery to coagulate the left common carotid artery (CCA), then we returned the pups to the cages for $60 \mathrm{~min}$, then put the cages in a designated square space to expose the mice to hypoxia for $2 \mathrm{~h}$. The hypoxic environment is a mixture of $8 \%$ oxygen and $92 \%$ nitrogen. When the exposure to hypoxia was complete, the HIE model was established. Sham controls underwent anesthesia and the left CCA was exposed as in the HI group, but without ligation or exposure to hypoxia.

GSPE (Meilun Biological, Dalian, China) was dissolved in PBS at a concentration of $1.25 \mathrm{mg} / \mathrm{ml}$. The GSPE+HI group was administered a dose of $30 \mathrm{mg} / \mathrm{kg}$, which was administered intraperitoneally and administered for $20 \mathrm{~min}$ before surgery. The HI group was given the same dose of PBS.

\subsection{Immunofluorescence Staining}

We deeply anesthetized the pups with $4 \%$ chloral hydrate and transcardially-perfused with $4 \%$ paraformaldehyde on the third day after surgery. The brain tissues were dissected and fixed in paraformaldehyde solution overnight. After gradient dehydration with ethanol and xylene, the brain tissues were prepared as wax blocks and cut into $5-\mu \mathrm{m}$ thick sections. Brain tissue sections were selected for subsequent immunofluorescence experiments. After deparaffination, the sections were placed in $0.01 \mathrm{M}$ citrate buffer and repaired using A

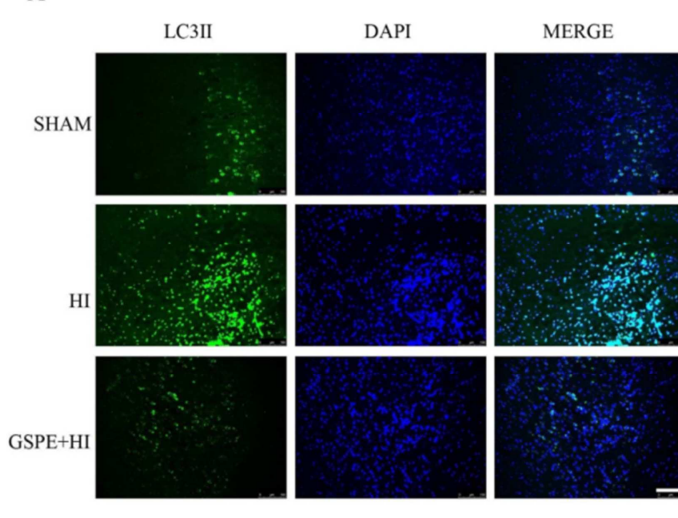

Figure 1. GSPE reduced the expression of autophagic-related proteins (LC3II and beclin1) 3 days after HI injury. a microwave for $20 \mathrm{~min}$. After blocking with goat serum for $1 \mathrm{~h}$, the sections were incubated at $4^{\circ} \mathrm{C}$ overnight with the following primary antibodies: anti-lc3II (1:800); and anti-beclin1 (1:800). Subsequently, the sections were incubated with the corresponding fluorescent secondary antibody. Brain slices were imaged with a fluorescence microscope.

\subsection{Western Bloting}

On the third day after surgery, the pups were deeply anesthetized with $4 \%$ chloral hydrate, and the ipsilateral brain tissues were removed. After adding RIPA lysate and PMSF (RIPA lysate: PMSF=100:1), the brain tissues were thoroughly ground with a tissue grinder, further centrifuged in a $4^{\circ} \mathrm{C}$ freezing centrifuge for $20 \mathrm{~min}(12,000 \mathrm{rpm})$, and the supernatant liquid was obtained for the next experiment. The above experimental samples were quantified using a BCA protein quantification kit (Beyotime Biotechnology, Shanghai, China). Specific operations followed the instructions of the reagent supplier. Equal amounts of protein $(20 \mu \mathrm{l})$ were loaded and separated on a SDS-PAGE gel and transferred onto PVDF membranes. Blots were probed with anti-LC3II (1:1500) and anti-beclin1 (1:1500), and the corresponding secondary antibody was used to further label the proteins. For the Western blotting method, $\beta$-actin $(1: 2000)$ was used as an internal control protein. The results were processed and analyzed using Image J software and SPSS 19.0 software.

\subsection{Statistical Analysis}

All the data were presented as the mean \pm SEM. Statistical significance was assessed by one-way analysis of variance (ANOVA), followed by a Bonferrioni test if more than two groups were involved. All statistical analyses were performed using SPSS.19.0 software and Graphpad Prism (version 4.0). $\mathrm{P}$ value $<0.05$ was considered as statistically significant.

\section{Result}

GSPE pre-treatment inhibits the expression of autophagic-related proteins in brains of the HIE model B

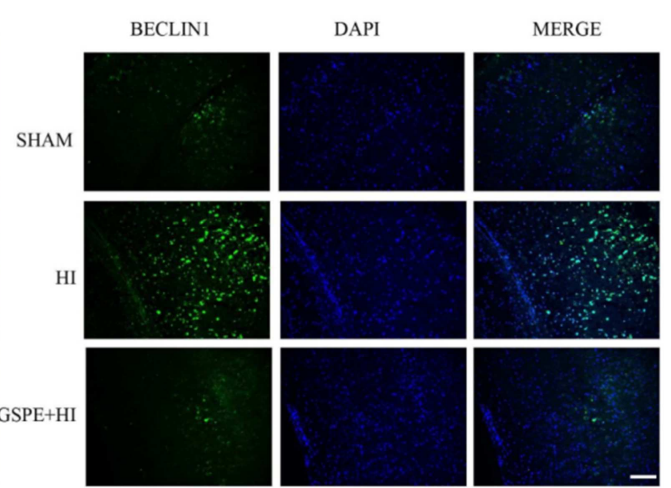

Representative immunofluorescence staining of LC3II and beclin1 (green) in ipsilateral brain tissue (A and B), DAPI-labeled the nucleus (blue). Compared to the sham group, the expression of LC3II and beclin1 increased in the HI group in ipsilateral brains. In GSPE+HI group, the expression of LC3II and beclin1 decreased more than the HI group. Scale bar $=100 \mu \mathrm{m}$. 
To investigate the effect of GSPE pre-treatment on autophagic protein expression, we performed immunofluorescence staining for autophagy-related proteins (LC3II and beclin1) in ipsilateral brains tissues (Figure 1A, 1B). On day 3 after HI injury, we observed a significant increase in the expression of LC3II and beclin1 proteins in the HI group. Compared with the HI group, expression of LC3II and beclin1 proteins was significantly reduced in the GSPE+HI group.

A

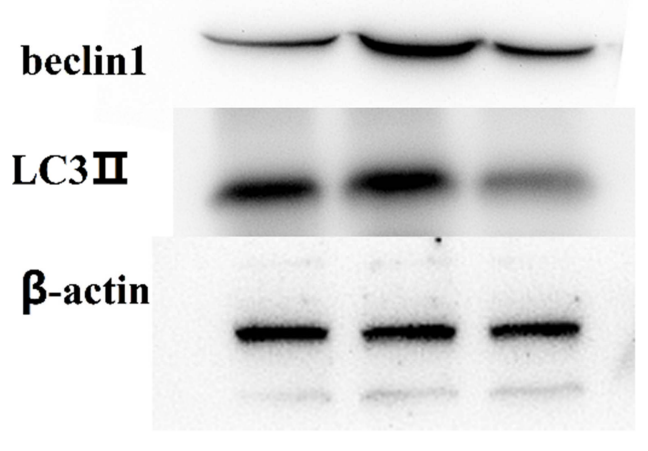

To further validate the effects of GSPE on autophagy in the HIE model, we used Western blot to quantify the expression of autophagosome marker proteins (LC3II and beclin1). Representative Western blots revealed that the expression of LC3II and beclin1 in the HI group was significantly increased compared with the sham group. Pre-treatment of GSPE reversed the increasing level of LC3II and beclin1 (Figure $2 \mathrm{~B}$, C). The above data indicate that the neuroprotective effect of GSPE on HI injury is achieved by inhibiting autophagy.

B

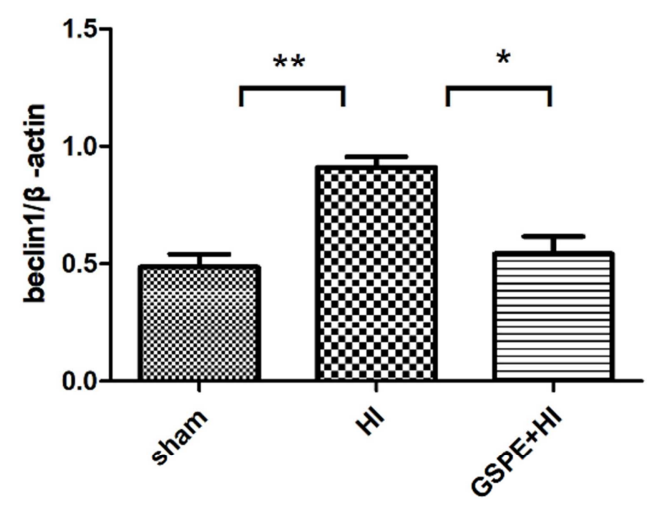

C

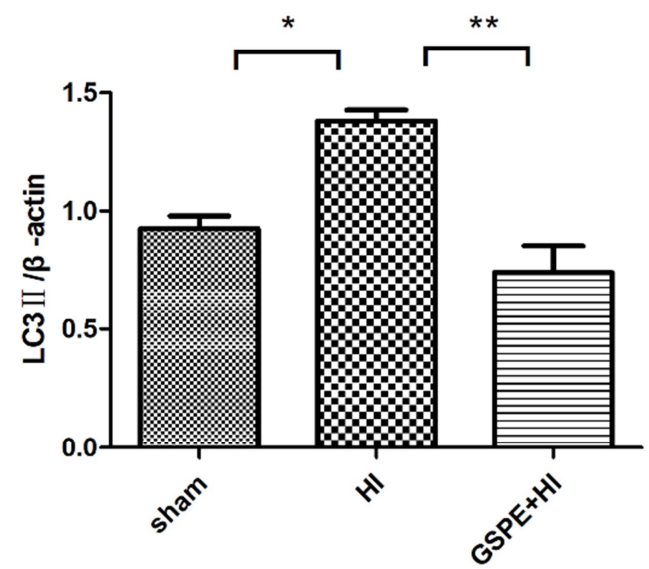

Figure 2. GSPE pre-treatment decreased the expression of autophagy-related proteins after HI injury.

Representative Western blots (A) and quantification data (B) (C) of beclin1 and LC3II are shown. In the HI group, the expression of beclin1 and LC3II was higher than the sham group. Compared to the HI group, the mean percentage of LC3IIand beclin1 was significantly induced in the GSPE+HI group. All calculations are normalized to $\beta$-actin. Values represent the mean $\pm \mathrm{SEM},{ }^{*} \mathrm{P}<0.05, * * \mathrm{P}<0.01$. One-way ANOVA followed by the Bonferroni test.

\section{Discussion}

Neonatal hypoxic-ischemic brain damage is caused by hypoxia and asphyxia during the neonatal perinatal period [12]. The pathologic events of HIE include apoptosis, autophagy, oxidative stress, and inflammation [13]. The type of brain injury depends on the degree of hypoxia, the duration of hypoxia, and the process of brain maturation [14]. At present, the clinical treatment of HIE involves hypothermia therapy [15], but this therapy only has efficacy on mild HIE injury; the effect is not significant in the treatment of moderate-to-severe HIE injury [16]. Therefore, finding a drug that can target HIE injury has become a difficult problem we need to solve.

Autophagy is an intracellular degradation process and a cytoprotective response of cells in extreme environments, such as starvation, hypoxia, and physical stimulation [17]. Autophagy can be converted into the energy needs by removing metabolic substances from the body and damaged 
organelles. Therefore, autophagy is irreplaceable in the process of cell survival [18]; however, the role of autophagy in HIE injury remains controversial. Some scholars believe that autophagy has a neuroprotective effect on $\operatorname{HIE}[19,20]$, and HIE injury can induce autophagy, which increases after administration; however, some scholars do not agree with this view. Although autophagy was increased 48-72 h after HIE, severe hippocampal injury was observed simultaneously. We cannot rule out the possibility that an increase in LC3 II/beclin 1 may by caused by inadequate autophagy.

In our study the data showed that the expression of autophagy increased significantly when HIE injury occurred, and we observed a significant decrease in the autophagy-related proteins after drug intervention with GSPE. This finding is in contrast to the results of others. We speculate that the possible reasons for this lack of consensus include the following: different sampling time; different degrees of hypoxic-ischemic injury; different varieties of pups; and different building model methods.

In addition, this study had limitations. The gold standard for detection of autophagy is the direct observation of autophagosomes in an electron microscope. Although LC3 IIand beclin1 are markers of autophagy, because LC3II will also be degraded by lysosomes, we need to use a combination of lysosomal inhibitors and protein markers to detect autophagy.

In summary, this study confirmed the protective effect of GSPE on the HIE mice model from the perspective of autophagy, and provides a theoretical basis for the future targeted therapy for neonatal hypoxic-ischemic brain damage.

\section{Conclusion}

In general, from qualitative and quantitative perspectives, we used immunofluorescence and western blotting to analyze the changes of autophagy-related proteins (LC3 IIand beclin1) before and after GSPE pretreatment. Our study initially demonstrated that GSPE exerts neuroprotective effects on neonatal mice models of hypoxic-ischemic encephalopathy by inhibiting autophagy.

\section{Acknowledgements}

This project was financially supported by the Natural Science Foundation of Guangdong Province (2018A030313579), the Science Foundation of Guangdong No. 2 Provincial People's Hospital for Youth (grant no. YQ2017-001), the Science and Technology Programs in Educational Commission of Guangdong Province (2016), the Innovative and Efficient Projects of Guangdong Pharmaceutical University (2016), and the Guangdong Province Innovation and Entrepreneurship Training Program for University Students (grant no. 201710573046).

\section{Competing Interests Statement}

The authors declare that they have no competing interests.

\section{References}

[1] Liu X M, Feng Y and Li A M (2014). Nerve protective effect of Baicalin on newborn HIBD rats. Asian Pac J Trop Med 7: 806-810.

[2] Dai Y, Li W, Zhong M, Chen J, Cheng Q., Liu Y, and Li T (2017). The paracrine effect of cobalt chloride on BMSCs during cognitive function rescue in the HIBD rat. Behav Brain Res 332: 99-109.

[3] Xu B, Xiao A, Chen W, Turlova E, Liu R, Barszczyk A, Sun C, Liu L, Tymianski M, Feng Z and Sun H (2016). Neuroprotective effects of a PSD-95 inhibitor in neonatal hypoxic-ischemic brain injury. Mol Neurobi, 53: 5962-5970.

[4] Liu G, Li Z G and Gao J S (2017). Hypothermia in neonatal hypoxic-ischemic encephalopathy (HIE). Eur Rev Med Pharmacol Sci 21: 50-53.

[5] Rao R, Trivedi S, Distler A, Liao S, Vesoulis Z, Smyser C, and Mathur A M (2019). Neurodevelopmental Outcomes in Neonates with Mild Hypoxic Ischemic Encephalopathy Treated with Therapeutic Hypothermia. American journal of perinatology. doi: 10.1055/s-0038-1676973.

[6] Liu B, Zhang H, Tan X, Yang D, Lv Z, Jiang H, Lu J, Baiyun R and Zhang $Z$ (2017). GSPE reduces lead-induced oxidative stress by activating the Nrf2 pathway and suppressing miR153 and GSK-3 $\beta$ in rat kidney. Oncotarget 8: 42226-42237.

[7] Niu L, Shao M, Liu Y, Hu J, Li R, Xie H, Zhou L, Shi L, Zhang $\mathrm{R}$ and Niu Y (2017). Reduction of oxidative damages induced by titanium dioxide nanoparticles correlates with induction of the Nrf2 pathway by GSPE supplementation in mice. Chem Biol Interact 275: 133-144.

[8] Wei M, Guo F, Rui D, Wang H, Feng G, Li S, and Song G (2018). Alleviation of Arsenic-Induced Pulmonary Oxidative Damage by GSPE as Shown during In vivo and In vitro Experiments. Biol Trace Elem Res 183: 80-91.

[9] Muzzi M, Buonvicino D, Urru M, Tofani L and Chiarugi A (2018). Repurposing of dexpramipexole to treatment of neonatal hypoxic/ischemic encephalopathy. Neurosci lett 687: 234-240.

[10] Santa-Maria I, Diaz-Ruiz C, Ksiezak-Reding H, Chen A, Ho L, Wang J and Pasinetti G M (2012). GSPE interferes with tau aggregation in vivo: implication for treating tauopathy. Neurobiol Aging 33: 2072-2081.

[11] Taniguchi H and Andreasson K (2008). The hypoxic ischemic encephalopathy model of perinatal ischemia. JoVE 21: e955-e955.

[12] Liu X M, Feng Y and Li A M (2015). Effect of G-CSF and TPO on HIBD in neonatal rats. Asian Pac J Trop Med 8: 132-136.

[13] Fang M, Jiang H, Ye L, Cai C, Hu Y, Pan S, Li P, Xiao J and Lin Z (2017). Metformin treatment after the hypoxia-ischemia attenuates brain injury in newborn rats. Oncotarget 8: 75308-75325.

[14] Bano S, Chaudhary V, and Garga U C (2017). Neonatal hypoxic-ischemic encephalopathy: A radiological review. J Pediatr Neurosci 12: 1-6. 
[15] Lee B S, Jung E, Lee Y, and Chung S H (2017). Hypothermia decreased the expression of heat shock proteins in neonatal rat model of hypoxic ischemic encephalopathy. Cell Stress and Chaperones 22: 409-415.

[16] Nair J and Kumar, V (2018). Current and Emerging Therapies in the Management of Hypoxic Ischemic Encephalopathy in Neonates. Children 5: 99.

[17] Zheng Z, Zhang L, Qu Y, Xiao G, Li S, Bao S, Lu R and Mu D (2018). Mesenchymal stem cells protect against hypoxia ischemia brain damage by enhancing autophagy through BDNF/mTOR signaling pathway. STEM CELLS 36: 1109-1121.
[18] Hara T, Nakamura K, Matsui M, Yamamoto A, Nakahara Y and Suzuki-Migishima R (2006). Suppression of basal autophagy in neural cells causes neurodegenerative disease in mice. Nature 441: 885-889.

[19] Ye L, Feng Z, Doycheva D, Malaguit J, Dixon B, Xu N, Zhang J and Tang J (2018). Cpg-odn exerts a neuroprotective effect via the tlr9/pampk signaling pathway by activation of autophagy in a neonatal hie rat model. Experimental Neurology 301: $70-80$.

[20] Li P, Hao L, Guo YY, Yang G L, Mei H, Li X H and Zhai Q (2018). Chloroquine inhibits autophagy and deteriorates the mitochondrial dysfunction and apoptosis in hypoxic rat neurons. Life Sciences 202: 70-77. 